\title{
THE DIAGNOSIS AND TREATMENT OF TUBERCULOUS CERVICAL ADENITIS
}

\author{
By Basil Armstrong, M.B., B.S. \\ Medical Superintendent of The Royal Sea Bathing Hospital, Margate
}

Tuberculous glands of the neck may be divided clinically and pathologically into three main groups, the first two of which are common, the third rather infrequent.

(a) The first is that in which the glandular enlargement is consecutive to a tuberculous infection of the tonsils or nasopharynx, more rarely to an infected tooth socket, to a localized tuberculous infection of the skin of the face (lupus) or to a tuberculous lesion of the eye or ear.

In these cases the appropriate gland, draining the structure primarily involved, first enlarges, and from it the infection spreads to other glands in the cervical chain, but in general in a downward direction, though lateral spread may occur even to the extent of involving glands on the opposite side of the neck. Tonsillar and nasopharyngeal infections are by far the commonest, and in their case the gland first involved is situated high up in the cervical chain just behind the angle of the jaw. In severe cases, the spread may be such as to involve the whole length of the deep cervical chain as well as submaxillary, submental and even occipital glands, but the important feature is that it takes place mainly in a downward direction.

It is frequently stated that the tubercle bacillus can pass through an intact mucous membrane without producing a lesion at its point of entry and can reach and involve a gland draining the region concerned, and there set up a primary tuberculous infection. This statement is most often made in regard to the infection of cervical and mesenteric glands.
I do not believe it to be true, but believe that a sufficiently careful search would reveal the primary infection to be at the point of entry, be it tonsil or intestinal mucosa. The infection is therefore exactly on all fours with the primary complex of lung and hilar infection.

(b) In the second important group, the lymphatic spread is from below upwards, the first glands of the neck observed to be involved being usually in the supraclavicular region. The involvement is consecutive to an extensive mediastinal gland tuberculosis, in its turn resulting from a lung infection of variable intensity, and may ultimately attack a large number of glands in the neck, but the spread takes place mainly from below upwards, and the glands which are visible and palpable are, it must be remembered-and this is an important point in deciding upon a line of treatment-probably only a small proportion of the whole number affected. They are, as it were, the apples on the uppermost branches of the tree, the lower branches of which are concealed from sight, but are doubtless also heavily laden with fruit. (Fig. I.)

(c) A third type of tuberculous cervical glands probably results from a haematogenous spread. These cases, which are somewhat uncommon are characterized by the rapid and almost simultaneous involvement of a large number of glands throughout both sides of the neck and of the axillae likewise. The glands tend to be large, fleshy, less prone to become adherent to their fellows and less liable to caseate and soften than those of the two pre- 
ceding groups-in fact, they approximate to the type of glands seen in Hodgkin's disease and may be difficult to distinguish from them without a biopsy.

Tuberculous glands occur mainly in children of all ages and in adolescents, although no age is immune. The greatest frequency is probably in children between the ages of five and ten, and falls steadily as age increases, becoming relatively uncommon after the age of 20 . The larger proportion of cases falling into the first group-i.e. those due in the main to tonsillar and nasopharyngeal infections-occurs in children. The second and third groups, which are, necessarily associated with a mediastinal gland tuberculosis of some chronicity, are more likely to be seen in adolescents and young adults. Males and females are about equally affected.

The infection may be either by the bovine or human strain of the bacillus. In the first group, bovine infection probably predominates, though to a less degree than is usually stated. Of the two other groups, the infection is much the most frequently by the human strain of the bacillus. .

Diagnosis of Tuberculous Adenitis of the neck. The diagnosis is made largely on the history of a slowly occurring, usually painless, enlargement of the glands of the neck. To differentiate between the cases falling into the two common groups, it is important, where the disease is already widespread throughout the neck, to discover whether enlarged glands were first noticed in the upper cervical group or in the supraclavicular region ; in earlier and less extensive cases, the question presents no difficulty.

In the descending type of infection, the disease is usually apyrexial, or almost so, and the general health little affected; in the ascending type, constitutional symptoms are more likely to be present: and there may be evening pyrexia of $100^{\circ}$ to $102^{\circ}$ with pallor, loss of weight and anaemia.

In the class, in which an extensive haematogenous involvement occurs, the general disturbance is again likely to be severe.

The glands will. present a different appearance according to the stage at which they are first seen.

In the early stages they are usually some- what fleshy and elastic, and not adherent to one another or to surrounding structures. Later, however, periadenitis occurs, the earlier infected glands tend to coalesce into a considerable mass, many of them becoming firm and tending to caseate, while the more recently infected glands, lying beyond the periphery of the mass, are still discrete, soft and only moderately enlarged.

Later the caseous glands may break down and abscess formation result. The abscess may be superficial 'ab initio, as where a superficial gland is concerned, or a deeply seated abscess may send a prolongation to the surface; which burrows its way through the muscle and fascia and eventually becomes subcutaneousthe so-called ' collar stud' abscess. As the abscess approaches the surface, the skin becomes thin, distended and bluish-red in colour, and frequently ruptures, with the development of a sinus, sometimes complicated by the occurrence of a surrounding scrofuloderma.

In the case of cervical adenitis secondary to a mediastinal infection, a radiograph will often show enlarged mediastinal glands, with or without a recognizable pulmonary lesion.

\section{Differential Diagnosis}

(I) Simple adenitis due to oral or skin sepsis, as in the presence of a focus of sepsis in the tonsils or teeth, of impetigo or of pediculosis of the scalp is usually easily recognized. It runs a more acute course, with fever, pain and some general malaise. The glands usually recede when the primary focus of infection is treated, but may soften, point and discharge pus.

(2) Streptococcal adenitis, due to a streptococcal tonsillitis, has an acute onset with sore throat and high temperature and considerable constitutional disturbance. The glands are hard and tender at first. They usually resolve with the disappearance of the causal infection, but may suppurate or may remain swollen for a long time, in which case, differentiation from a tuberculous adenitis may be difficult. However, the history of an acute onset and a tuberculin test, if negative, will help in arriving at a correct conclusion.

(3) Hodgkin's Disease (Lymphadeonoma) has a gradual onset, and glands in other parts of the 


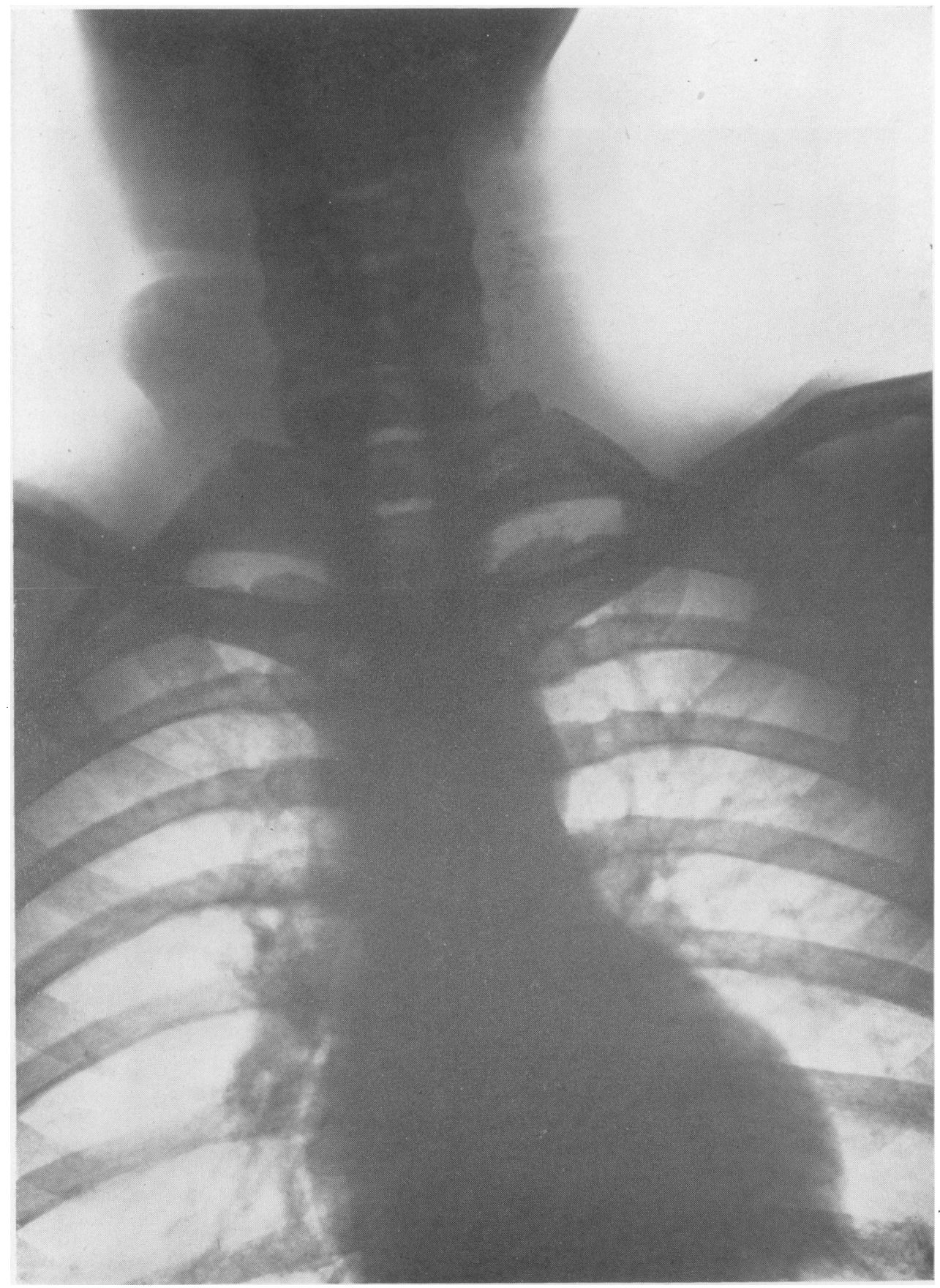

Fig. I. Tuberculosis of Mediastinal Glands. 


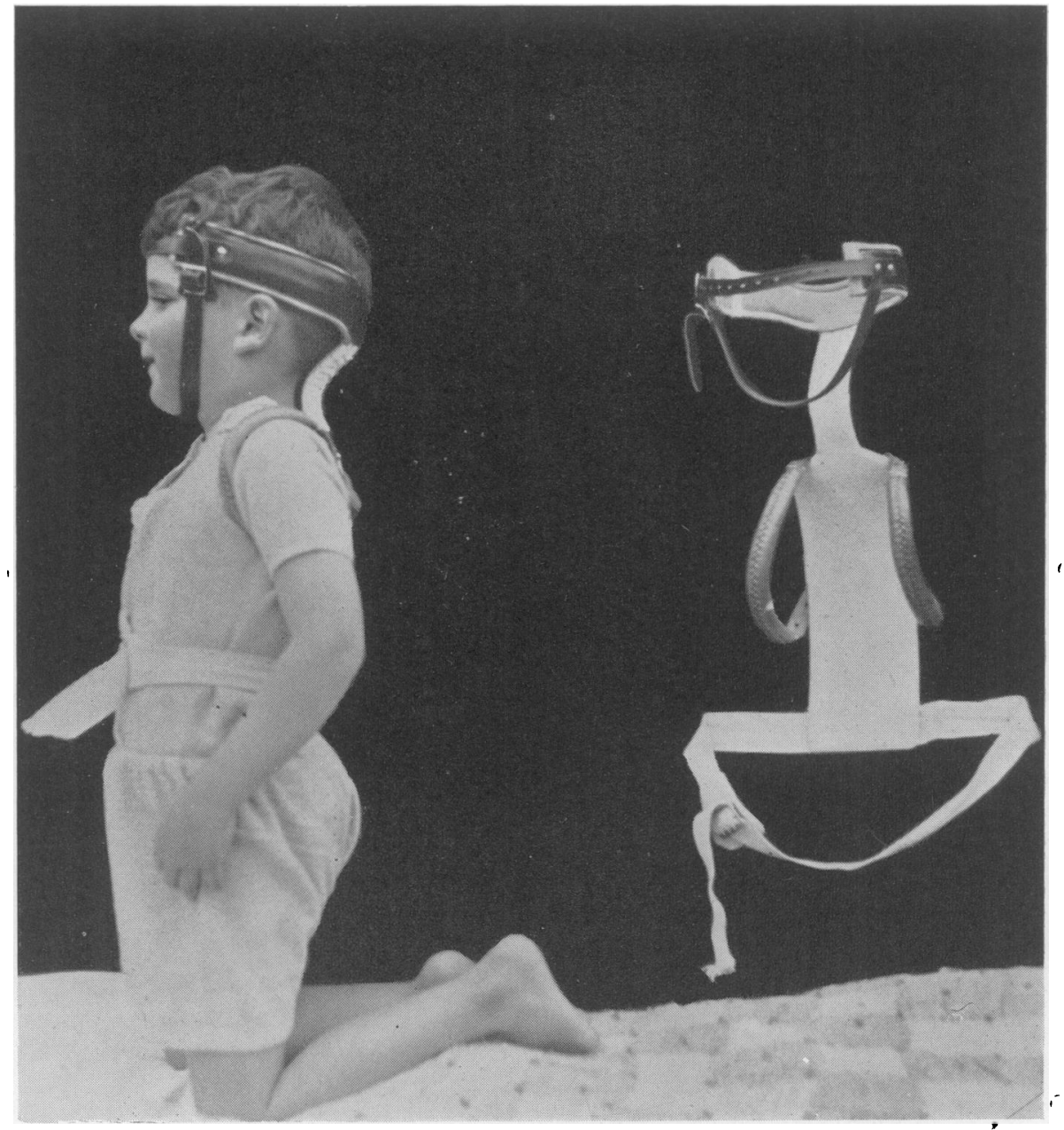

Fig. 2. Type of neck splint described in text.

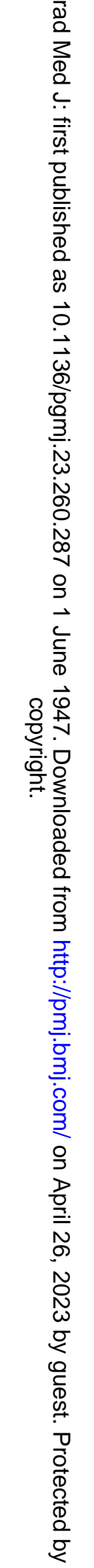




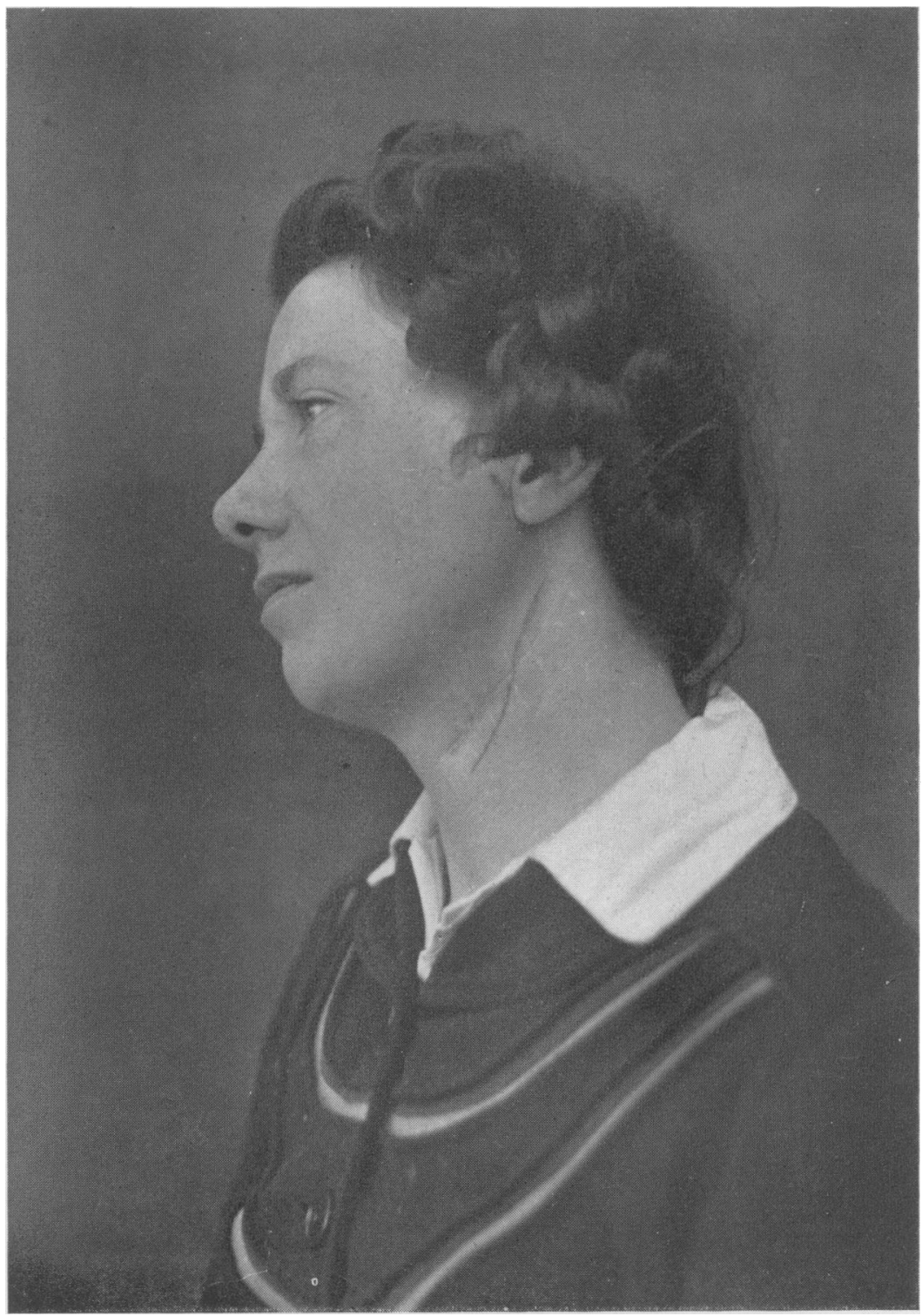

Fig. 5. A bad scar, the presence of which was advertised by stitch marks due to too late removal of sutures, lying in front above the level of the natural crease (marked by ink line). As a result of this the infra-mandibular branch of the cervical division of the facial nerve was damaged, producing a permanent weakness of the muscles of the corner of the mouth as shown in the photograph. 


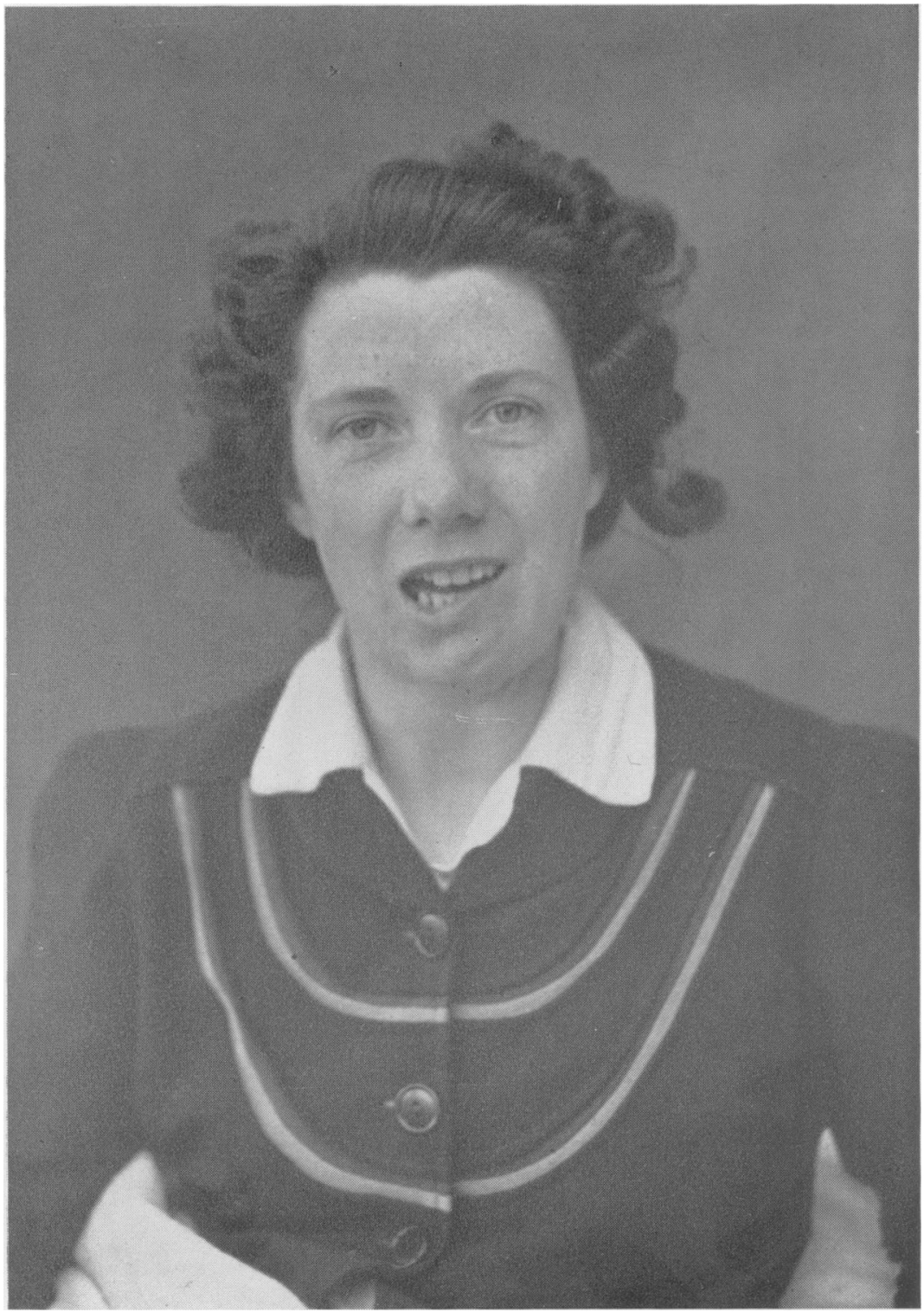

Fig. 6. Permanent weakness of the muscles of the left corner of the mouth resulting from damage at the time of operation to the infra-mandibular branch of the cervical division of the Facial Nerve. The placing of the anterior extremity of the incision is at too high a level-in this case at about $\frac{3}{1}$ in. above the upper natural crease. 
body may be enlarged also. In its later stages, splenic enlargement may be present, and there is associated anaemia with increased cosinophil count and often some degree of leucocytosis. The glands are large, discrete, elastic and nontender. They do not tend to fibrose or caseate. The condition of tuberculous glandular infection described above as being of probably haematogenous origin, may closely resemble Hodgkin's disease. The tuberculin reaction, if negative, may help in diagnosis, but the removal of a gland for biopsy will often be necessary.

(4) Lymphatic Leukaemia shows a picture of severe illness with widespread lymphatic enlargement, high fever, splenic enlargement and a characteristic blood picture.

(5) Lymphosarcoma-a condition very rare in children-may suggest a tuberculous infection, but its rapidly progressive course will usually suggest the advisability of the performance of a biopsy.

\section{Treatment}

Many cases of tuberculous cervical adenitis, particularly those coming under treatment in their early stages, will make a spontaneous recovery. The enlarged glands, which have not gone on to caesation, diminish in size and may cease to be palpable, while some large caseous glands may, in the course of time, become encapsulated and fibrose and shrink to such an extent as no longer to constitut $\epsilon$ any serious danger. Such glands may eventually calcify completely or in part. But the process where caseous glands are concerned is slow and uncertain, and for a long time a danger will persist of their softening and forming abscesses with a possibility of rupture and a formation of ugly scars.

In general, all cases of whatever type should, at first, be given a course of from three to six months of constitutional treatment.

In the cases falling into the first group-i.e. those in which the glandular involvement is secondary to an infection of the tonsils or nasopharynx, of the eye (e.g. tuberculous conjunctivitis), of the ear (e.g. tuberculous otitis media or externa), or of the skin, the appropriate treatment of the primary focus is of paramount importance.

I have no hesitation in saying that, in the case of tonsillar or nasopharyngeal infection, the tonsils and adenoids should be removed as soon as the patient's general condition permits, the only exception being where a glandular abscess is present or is threatening, in which case it may be well to postpone tonsillectomy until the abscess has been successfully dealt with.

Constitutional treatment should be carried out, whenever possible, at the seaside or in the country. If possible the patient should be absolutely at rest and confined to bed. Heliotherapy and open-air treatment is of great value; the diet should be generous; extra milk should be given, and in many cases the administration of halibut or cod liver oil will be beneficial.

The use of a splint to restrict movement of the head and neck is helpful in all cases, particularly in disease of the upper cervical glands.

A simple splint, easily made by anyone possession a small amount of mechanical skill, is illustrated. It consists, in the main, of a strip of duralumin sheet, running up vertically behind the upper thorax and neck, some 5 in. or more wide behind the thorax, and 2 in. or more wide behind the neck, and extending from waist to occiput. This is bent to fit the curves of the upper thorax, the back of the neck and head, and is reinforced by means of a strip of duralumin bar riveted to it. At its upper end it carries a 5 in. strip of duralumin, extending forwards on either side of the head in the form of a horseshoe, encircling the posterior two-thirds of the head. The anterior one-third is closed by means of a leather strap running across the forehead, and another strap runs from side to side below the chin. The splint is anchored to the thorax by means of padded axillary straps and a wide webbing band around the waist. A thin layer of white felt is glued to the surface of the splint in contact with the body and head, and the whole is completed by a covering of unbleached calico. (Fig. 2.)

Thus treated, many cases of cervical adenitis will improve to such an extent as to make the consideration of an operative removal of the diseased glands unnecessary.

A proportion will remain, however, in which the size of the glands and their chronicity will 
make it unlikely that they will heal and absorb spontaneously within a reasonable time, and of these cases those falling into the first group should be treated by the radical excision of all the enlarged glands. By this means, a complete cure of the condition may be obtained with the reasonable prospect of insignificant disfigurement of the neck, if any at all.

At this point, it is worth while to discuss the desirability or otherwise of operation upon the cases falling into groups (b) and (c), viz., those which have either an ascending lymphatic infection from a group of intrathoracic tuberculous glands, and those in which the glandular involvement is due to a haemic spread. Both groups are bad subjects for extensive surgery, particularly the latter.

In the former group, it is likely that glands, here and there, will break down and form abscesses. In general, these should be treated as conservatively as possible. The widest excision practicable will only remove a small proportion of the diseased glands, the operation is likely to be severe and will perhaps be sufficient to upset the resistance of the patient which we have been building up laboriously by months of constitutional treatment. Treat these cases, then, by aspiration, where possible, of their abscesses, and failing this by a small transverse incision with the evacuation of their contents and light curetting.

In parenthesis, it may be said that the aspiration of tuberculous abscesses of the neck is less successful than in the case of cold abscesses in most other situations. The pus is almost always very thick and a very largebored needle must be used and successive aspirations tend to become more and more difficult, the lumen of the needle being almost invariably blocked by tough fibro-caseous debris. However, the manoeuvre is always worth attempting in the case of large deepseated abscesses, in the hope that one or two aspirations will cause them to dry up; where the abscess has become superficial and the skin is stretched and discoloured, incision and evacuation is the best line of treatment, for in these circumstances even if the skin does not break down spontaneously a lumpy brownish semi-keloid scar results which is more conspicuous than the scar of the small incision.
If the skin over a superficial abscess breaks down and sloughs, as is not infrequently the case, this is not a major disaster. The removal of the contents of the deeper part of the abscess with a curette will usually be followed by the production of a flat ulcer with a base covered with short healthy granulations to which a small Thiersch graft may readily be caused to adhere and an excellent cosmetic result obtained.

In the case of the large fleshy glands resulting from a haemic spread, the temptation to operate must be sternly resisted-a temptation which is all the stronger because, in such cases, there is little tendency for the glands to caseate or to become adherent and their removal, therefore, is a matter of great simplicity. In the $f \in w$ cases, however, where, in my experience, operation has been performed, it has been poorly tolerated and an early and extensive recurrence has resulted. Moreover, in this class of case, given adequate and sufficiently prolonged constitutional treatment, the tendency for spontaneous absorption and resolution to occur is very marked.

Reverting, then, to the first group of cases, those in which the infection may be termed a descending one, it has been seen that, after a reasonable period of conservative treatment has been given, there will still be a proportion of cases in which large caseous glands remain and tequire operative removal. One advantage of a preliminary period of rest and constitutional treatment is that, in addition to the building up of the patient's general resistance, the periadenitis which causes the glands to become matted together and adherent to surrounding structures will have largely disappeared, and the operation is thereby rendered easier, and what is perhaps more important, any small seed-like glands which may be missed at operation are much less likely to enlarge subsequently, and to cause recurrences, than if surgical intervention had been undertaken, prematurely. The only exception to the rule of waiting for from three to six months is in the case of glands which are breaking down to form abscesses. The difficulty, already mentioned; of treating . these abscesses conservatively makes it worth while to operate early to avoid a disfiguring scar, even at a slightly greater risk of a recurrence. 


\section{Operation}

The object of operation in these cases is to achieve the fullest possible removal of diseased glands, with the smallest amount of disfigurement of the patient.

The success of the operation will be judged -and quite rightly - by the patient and his or her relatives, but the inconspicuousness or otherwise of the scar, and it is worth while going to some extra trouble to obtain an almost invisible scar.

It should not be necessary to point out that the incision should never run in the long axis of the neck parallel to the border of the sternomastoid muscle, as the resulting scar will always stretch widely and become very obvious, but it is insufficient to say that it should run transversely and parallel to the natural creases of the neck. It must actually coincide most meticulously with one of these natural creases of which there are usually two in the upper neck in the positions shown in the diagram. (Fig. 3.)

It will usually be found that one or other of these creases is conveniently placed in respect to the main mass of glands to be removed. If the glands are relatively few and situated high up under the anterior margin of the sternomastoid muscle, the upper crease may be chosen, but if they are numerous and extend for some distance down the course of the internal jugular vein, and particularly if they lie partly or largely in the posterior triangle of the neck, making it likely that the posterior border of the sternomastoid will have to be freed from its sheath to facilitate their removal, then the line of the lower of these creases affords the best access and offers two additional advantages, the first that the inframandibular branch of the cervical division of the facial nerve supplying the muscles of the corner of the mouth is less liable to be injured, the second that it is possible through this incision to clear away glands for a considerable distance down the neck; this may obviate the necessity of making a second incision except in the case of glands lying low down in the supraclavicular region. The disadvantage of using the lower crease is that it makes the freeing of the upper extremity of the gland mass, which may extend up almost to the base of the skull, more difficult and increases the risk of a troublesome

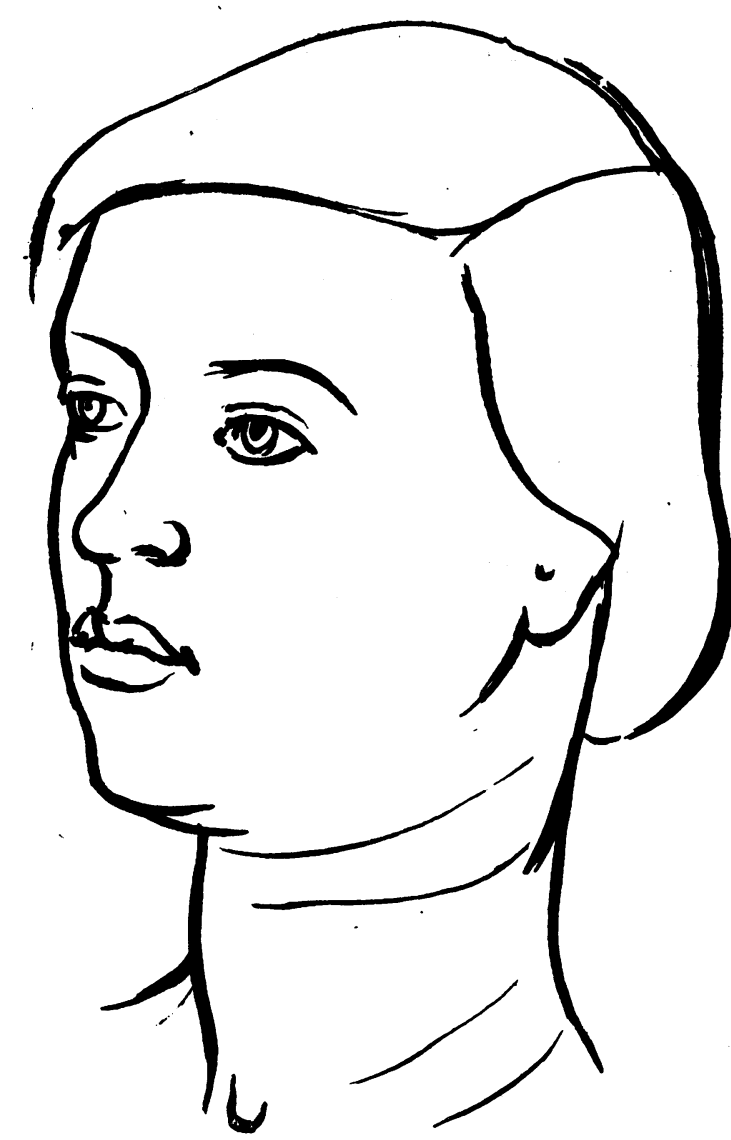

Fig. 3. The natural creases of the neck.

haemorrhage through the tearing of a small branch of the internal jugular in this very inaccessible situation.

If it is necessary to use a second incision low down in the neck it must, of course, follow the rule already laid down and must not only run transversely but must coincide closely with a natural crease.

As it is usually very difficult, particularly when the patient is anaesthetized, with the neck placed somewhat on the stretch, and in the presence of a large mass of glands, to define accurately the line of the natural creases of the neck, my own practice is always to spend a few minutes on the day proceeding the operation, in marking out, first with ink and then by needle scratch the exact line to be followed in incising the skin. At the same time three or four scratch marks are made at intervals, running at right angles to the line of the proposed incision. If these are made 24 


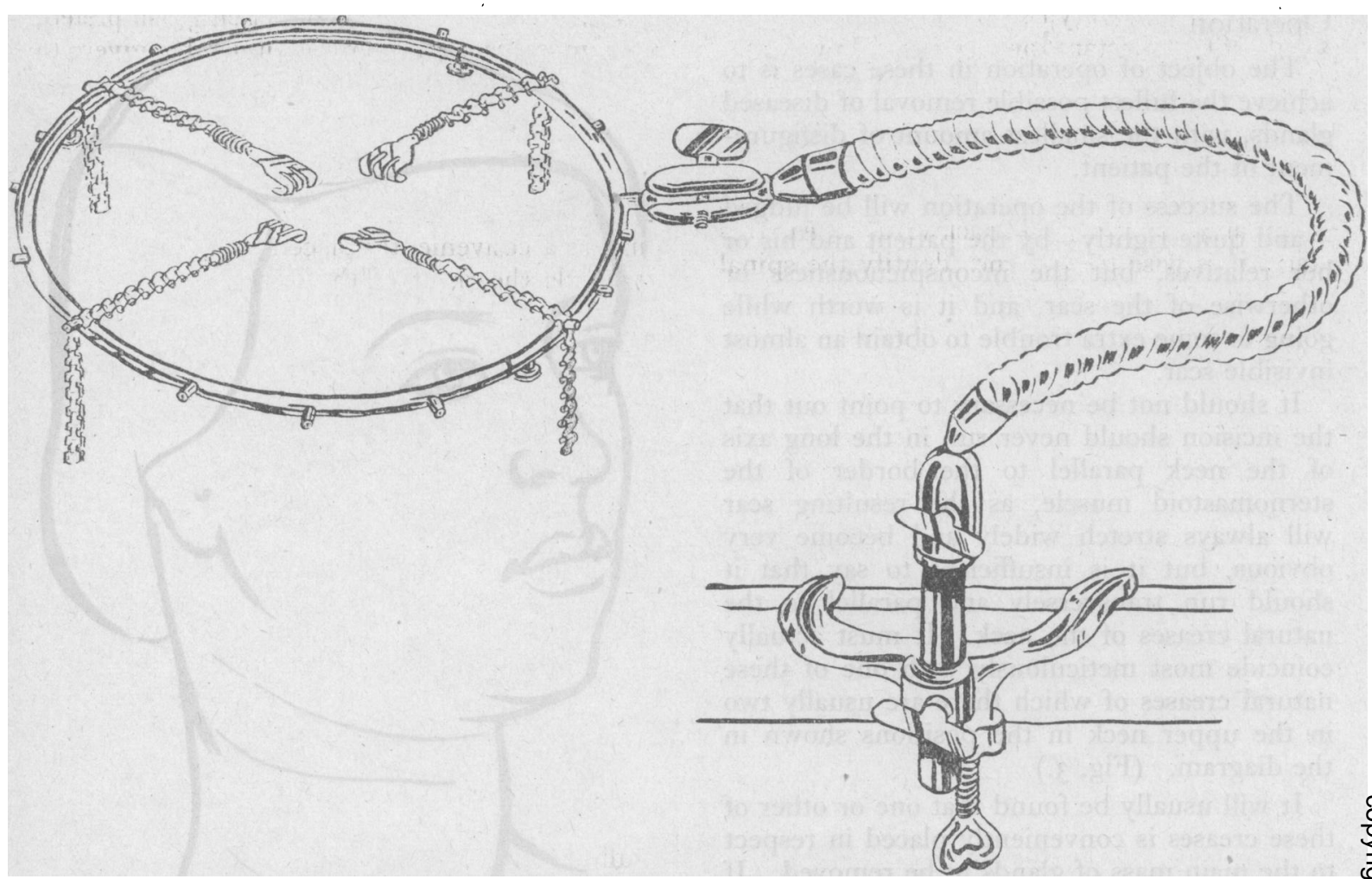

Fig. 4. The author's pattern of Self-Retaining Retractor. It consists of a metal ring, 14 ins. in diameter, held in position over the wound by a flexible metal arm to which it is attached by a universal joint. The flexible arm in its turn is fastened by a clamp to the edge of the table. The ring is made in three sections for ease of sterilization.

'The retractors, which are of various sizes, are attached through short coiled springs to lengths of 'ladder' chain, which can be hooked in appropriate positions over small blunt studs placed on the periphery of the metal ring.

They can, of course, be used to retract muscles as well as skin edges.

hours before operation, they are much more easily recognized when it comes to sewing up the skin, than if made as the operation starts, and accurate appositior, to the wound edges is greatly facilitated.

The anaesthetic should preferably be administered intratracheally. The patient lies on his back with his head turned to the sound side, and a small pillow is placed behind the shoulders and lower neck.

The incision is made in the predetermined line and must be of such a length as to allow good access to the region to be exposed, and no longer. It should not extend backwards behind the posterior border of the sternomastoid muscle further than is absolutely necessary, for in this region, scars easily tend to assume a slightly keloid character, and remain somewhat raised and reddened.
The incision extends through the skin and underlying fat. If the platysma is well developed, it should not be divided in this incision, but should be preserved and later split longitudinally in the course of its fibres, close to the anterior border of the sternomastoid. It can then be sutured with a few interrupted catgut stitches at the end of the operation, a procedure which diminishes the risk of a sunken scar after the removal of a large gland tumour. This depressed scar, when it occurs, is very conspicuous and may mar an otherwise excellent cosmetic result.

The skin flaps are undermined widely in all directions; the external jugular vein is divided between ligatures. Sinuses should not be excised but should be divided subcutaneously in the undermining of the skin flaps. A self retaining retractor to hold back 
the skin edges is very helpful and liberates both hands of the assistant for more useful purposes.

The platysma muscle having been split longitudinally, the anterior border of the sterno mastoid muscle is exposed over the full extent of the swelling formed by the gland mass and is retracted backwards. At this stage, it is wise to find and identify the spinal accessory nerve as it enters the deep surface of the muscle in order to preserve it from subsequent injury.

The enlarged glands will now be exposed and should be seized by suitable forceps and pulled backwards and the fascial tissue on their deep surface divided near the lower end of the operation area, where the internal jugular is most easily exposed. Dissection of the gland mass is most easily carried out by means of curved Mayo's scissors and swab pressure, and if the plare of dissection is kept as close as possible to the glands little difficulty is experienced in separating them from the vein, the tributaries of which should be clamped and tied as encountered.

The lower end of the mass being freed, dissection is continued upwards until the spinal accessory nerve is encountered. In order to clean and preserve it, division of the gland mass may be necessary. Keeping constantly in the areolar tissue plane immediately adjacent to the glands themselves and using scissor and gauze diss sction, the uppermost member of the chain will eventually be reached and the pedicle above this clamped and divided and the mass removed.

In those cases where the glands extend into the posterior triangle, it will be necessary, as has already been said, to free the posterior edge of the sternomastoid muscle, and here the spinal accessory nerve is in greater danger, as it lies more superficially. However, by keeping close to the glands themselves, and by exercising sufficient care and patience, it should be possible to avoid injury to this and to any other important structure.
The removal of the glands being completed, the most careful attention must be given to haemostasis, all divided vessels being ligatured with fine catgut. A twisted silkworm gut or fine rubber drain is inserted into the dead space under the sternomastoid muscle and is brought out at the anterior angle of the wound, unless a conveniently placed sinus had existed towards the front of the lower flap, when its orifice may advantageously be used for this purpose. This blood drain may be removed at the end of 24 hours. Haemostasis secured, the platysma is united by fine interrupted catgut sutures, and the skin closed with ophthalmic silkworm gut.

The greatest care must be exercised in securing perfect apposition of the skin edges. Childe's approximating forceps used by an assistant, thus allowing the surgeon the use of both hands in tying the sutures, is an excellent instrument in securing this end. The sutures must be numerous and must be placed close together-at a distance of not more than $\frac{1}{4}$ in. apart.

Closed in this way, the skin wound will be sufficiently soundly healed to allow the removal of the stitches on the third or fourth day. Left longer than this, stitch scars will result and nothing is more unsatisfactory than to have the presence of an almost invisible scar advertised by a double line of ugly white dots. (Figs. 5 and 6.)

The best dressing after operation is a strip of $I$ in. gauze ribbon soaked in Mastisol or Friar's Balsam and laid lengthwise over the wound. This is covered by layers of gauze and wool, held in position by a figure-of-eight bandage round the neck and skull. The gauze ribbon dressing, which allows the removal of the blood drain without disturbance, should be renewed atter the stitches are taken out.

A neck splint may, with advantage, be worn for three or four weeks atter the operation.

Convalescence should be of several months duration, preferably spent at the seaside or in the country. 